Methods We searched the literature for documents from the relevant organisations, and communicated with officials involved in the eradication efforts.

Results Some of the major lessons we identified for successful eradication efforts in this country were:

- Having a clear goal of achieving eradication.

- The disease/vector ecology and the points of intervention.

- Having effective tools available to achieve eradication

- Multidisciplinary funding from, and collaboration across, government agencies (agricultural, biosecurity, health) especially for hydatids control and SSM eradication.

- Having well-planned and well-resourced campaigns that could continue for $10+$ years.

- Having detailed surveillance systems to guide refinements of eradication efforts and to confirm success.

Conclusions New Zealand has been able to eradicate some zoonotic diseases and exotic disease vectors with well-designed campaigns. Lessons from such control efforts may be relevant to other countries (especially island nations) to protect human health, animal health and ecosystem health. Such tools, skills and collaborative efforts will be useful in facing the challenges of the future such as disease and vector eradication in the face of climate change.

\section{P1-371 WHAT'S POLITICS GOT TO DO WITH GLOBAL HEALTHCARE? A MULTILEVEL EXAMINATION OF INDIVIDUAL-PATIENT REPORTS OF HEALTH SYSTEM RESPONSIVENESS IN 45 LOW, MIDDLE AND HIGH INCOME}

doi:10.1136/jech.2011.142976f.63

\begin{abstract}
${ }^{1} \mathrm{M}$ Witvliet, ${ }^{*}{ }^{1} \mathrm{~A}$ Kunst, ${ }^{1} \mathrm{~K}$ Stronks, ${ }^{2} \mathrm{~T}$ Mahapatra, ${ }^{2} \mathrm{C}$ A Thompson, ${ }^{1,2} \mathrm{O}$ A Arah. ${ }^{1}$ Academic Medical Center (AMC), University of Amsterdam, Department of Public Health, Amsterdam, The Netherlands; ${ }^{2}$ UCLA School of Public Health, Los Angeles, California, USA
\end{abstract}

Introduction Patient responsiveness, often measured as patient experiences of healthcare, is a core dimension of health system functioning. Seldom is this investigated globally, with little known about the influence of politics on how different national systems are responsive to their patients. Therefore, we investigate (1) the associations between patient responsiveness and political factors, and (2) the extent to which health system inputs and outputs might further explain associations.

Methods World Health Survey data were analysed in 45 countries $(n=195891)$. Main outcomes included eight responsiveness indicators for both in-and out-patient experiences. Multilevel linear regression was used to assess associations of individual patients' responsiveness with policy metrics (ie, civil liberties, political rights), general development (ie, per capita GDP and female literacy), health system inputs (ie, health spending and human resource capital), and health system outputs, measured by maternal mortality. We also adjusted for individual-level socioeconomic factors.

Results Political factors are associated with patient responsiveness. Measured on a $0-5$ scale, political rights are more positively related to patient responsiveness than civil liberties. For example, betas and SE show that an increase in political rights indicates a 0.05 (SE 0.04) increase in attention, and a 0.02 (SE 0.05) increase in autonomy and choice, respectively. Associations with political factors are not greatly modified by general development indicators, nor by health system input or output.

Conclusion The manner in which a country is organised politically influences patient responsiveness. Strengthening a country's political infrastructure, might improve patient responsiveness, which may enhance health system functioning.

\section{P1-372 AN AGE-PERIOD-COHORT ANALYSIS OF MORTALITY ASSOCIATED WITH BACTERIAL DISEASES IN HONG KONG}

doi:10.1136/jech.2011.142976f.64

I 0 L Wong, * B J Cowling, G M Leung, C M Schooling. School of Public Health, The University of Hong Kong, Hong Kong SAR, Hong Kong

Introduction We aim to investigate longitudinal trends in bacterial disease related mortality in $\mathrm{HK}$ in order to describe how socioeconomic transition affects mortality due to infections and to provide generalised aetiological insights. We use one of the major bacterial disease related death, septicaemia, as an exemplar.

Methods We used local data on mortality due to septicaemia and mid-year population figures (1976-2005). We fitted Poisson ageperiod-cohort models on the age, period and cohort effects. We also looked for any possible difference in age, cohort or period effects by sex assessed from the model fit using the deviance information criterion (DIC).

Results Septicaemia-related deaths increased exponentially with age in both sexes, while there was a down turn in the period effects in both sexes (peaked in the 1991-1995 for females, and in the 1986-1990 for males). The birth cohort curves mainly had downward inflections in both sexes, however there was a steeper deceleration in women after 1945s, which was confirmed by the model fit as shown by the DIC.

Conclusion Sex difference in birth cohort effects might reflect changes which affect mortality risk due to septicaemia in a given birth cohort throughout their lifetime. The observed changes could be living conditions of the individual at different stages in life, and better immunity against bacteria in women born in HK but not in men. It is compatible with our hypothesis that the gonadotropic axis upregulation with better early living conditions enhance immunity in women but not men.

\section{P1-373 USING EPIDEMIOLOGICAL DATA TO INFORM SERVICE PLANNING: A LESSON FROM THE SCOTTISH HOME OXYGEN SERVICE}

doi:10.1136/jech.2011.142976f.65

R Wood, ${ }^{*}$ I Grant, M Bain. NHS National Services Scotland, Edinburgh, UK

Introduction Increasing demand for home oxygen in Scotland has resulted in a shortfall in the budget for this service. In 2009, discussions began to identify financial solutions. There was a lack of understanding about what was driving increasing demand and an assumption that this reflected inappropriate over-provision. A national home oxygen needs assessment was undertaken. This considered the epidemiology of underlying conditions to examine this assumption and contribute to planning.

Methods Analysis of routinely available data to examine the epidemiology of four conditions underlying home oxygen use: chronic neonatal lung disease, cystic fibrosis, chronic interstitial lung disease, and chronic obstructive pulmonary disease. Trends in risk factors, disease incidence, prevalence and mortality, and hospital admissions were examined.

Results The prevalence of all the conditions studied has increased in Scotland over recent years due to increased incidence, increased survival, more active case finding, and/or demographic changes. Trends towards considerably shorter hospital stays and hence more community based care were noted.

Conclusions The need for home oxygen is likely to continue to increase over coming years. It will encompass all age groups and a complex range of conditions. Initial planning assumptions anticipating reducing demand for home oxygen were unsound and adjusted accordingly. Routine data, particularly in linked format, is 